\title{
Amino acid transporter SLC38A3 promotes metastasis of non-small cell lung cancer cells by
}

\section{activating PDK1}

Yanhui Wang ${ }^{1,2, *}$, Li Fu ${ }^{1,2, *}$, Minqing Cui ${ }^{1, *}$, Yongbin Wang ${ }^{1}$, Yan $\mathrm{Xu}^{1}$, Molin $\mathrm{Li}^{2, \#}$, Jun $\mathrm{Mi}^{1, \#}$

1 Department of Biochemistry \& Molecular Cell Biology, Key Laboratory of Cell Differentiation

and Apoptosis of Chinese Ministry of Education, Shanghai Jiao Tong University School of Medicine;

2 Dalian Medical University Institute of Cancer Stem Cell;

*: these authors contribute equally to this study;

\#: Correspondence to: Jun Mi: jmei@sjtu.edu.cn; Molin Li: molin li@hotmail.com

Emails of co-authors: Yanhui Wang: wangyanh123@sina.cn; Li Fu: auojn@ 163.com; Minqing Cui:

13817211597@163.com; Yongbin Wang: wybzyp@ 126.com; Yan Xu: yan xu@sjtu.edu.cn; Molin Li:

molin li@hotmail.com; Jun Mi: jmei@sjtu.edu.cn

Running title: SLC38A3 promotes metastasis of NSCLC

Abstract: 161 words; Text: 4239 words (including references and figure legend); Figures: 4

Key words: SLC38A3, metastasis, NSCLC, Glutamine, PDK1 


\begin{abstract}
:
Tumor metastasis is a finely-tuned pathological process coupled to metabolic reprogramming that includes both glutamine and glucose. The solute carrier SLC38A3, a member of amino acid/ polyamine/ organocation (APC) superfamily, is an L-glutamine transporter. It is not clear whether SLC38A3 involves the metastasis of NSCLC (non small cell lung cancer). In this study, we reported that SLC38A3 was upregulated in metastatic NSCLC cells and its expression was correlated with prognosis of NSCLC patients. SLC38A3 overexpression promoted epithelial mesenchymal transition (EMT) and migration of HCC827 and A549 human lung adenocarcinoma cells, and accelerated tumor metastasis in mice. We found that SLC38A3 decreased the cellular concentrations of glutamine and histidine, and the deficiency of glutamine or histidine activated PDK1/AKT signaling that in turn, triggered NSCLC metastasis. SLC38A3 activated PDK1/AKT signaling and promoted metastasis of NSCLC through regulating glutamine and histidine transport, suggesting SLC38A3 as a potential therapeutic target for treatment of NSCLC.
\end{abstract}

\title{
Keywords:
}

SLC38A3, metastasis, NSCLC, glutamine metabolism, PDK1 


\section{Highlights:}

1. Expression of solute carrier SLC38A3 increased in metastatic NSCLC cells;

2. SLC38A3 exported glutamine/histidine in metastatic NSCLC cells, activated PDK1/AKT signaling;

3. SLC38A3 promoted epithelial - mesenchymal transition of NSCLC cells.

\section{Abbreviations:}

NSCLC, non-small cell lung cancer; EMT, epithelial - mesenchymal transition;

APC, amino acid/ polyamine/ organocation; SLC, solute carrier; NADPH, nicotinamide adenine dinucleotide phosphate-oxidase; GFP, green fluorescent protein 


\section{Introduction}

Metastasis is a multistep process starting with the dissemination of tumor cells from a primary site [1]. In healthy cells, deadhesion results in impaired glucose transport and subsequent apoptosis because of an energy deficit [2]. Cancer cells depend on anchorage-independent survival for migration from a primary site to target organs [3]. Metastasis-related proteins thus act to promote tumor cell migration, not only proliferation [4], and in addition to anchorage -independent survival, the metastasis-related proteins induce metabolic changes that are distinct from the Warburg effect $[1 ; 5 ; 6]$.

In addition to glucose, amino acid and lipid metabolism may also undergo significant change during metastasis. For example, hyperactivation of glutaminolysis in cancer cells generates NADPH for participating anabolic reactions that allow glucose carbons to be used for nucleotide, lipid and protein biosynthesis via ribose-5-phosphate or fatty acids as intermediate metabolites [7; 8]. In advanced grades of glioblastoma, increased concentration of glutamate and glutamine in the extracellular fluid has lead to consideration as extracellular signaling molecules that facilitate local spread of advanced grades of glioblastoma $[9 ; 10 ; 11 ; 12]$, implicating glutamine and glucose metabolism in the process of tumor metastasis. However, it is not clear about how glutamine metabolism is regulated in metastatic cancer cells.

With 395 proteins organized into 52 families, the solute carrier (SLC) transporters comprise the second largest group of membrane proteins in humans $[13 ; 14]$. Sequence profile analysis has 
revealed that 24 of the SLC families belong to three Pfam clans [15], MFS, APC and CPA/AT [16]. The SLC38, SLC32, and SLC36 families, are the only known members of the amino acid/polyamine/organocation (APC) superfamily [16]. The SLC38 family members are also known as sodium-coupled neutral amino acid transporters (SNATs), and have been further characterized as System N or System A transporters [17]. SLC38A3 (SNAT3), a member of the System N subfamily, has a preference for L-glutamine, L-histidine, L-alanine and L-asparagine [18]. SLC38A3 may mediate cellular glutamine efflux when the $\mathrm{H}^{+}$gradient is reversed, and presumably would effectively only transport glutamine down its chemical potential gradient when tumor cells detach from a primary site, since $\mathrm{Na}^{+}$-coupled glutamine efflux would have to overcome an unfavorable electrochemical potential gradient $[18 ; 19 ; 20]$. However, there is little evidence of SLC38A3 involvement of metastasis in NSCLC (non small cell lung cancer).

In this study, expression profiling data from a four-dimensional (4D) tumor model revealed that SLC38A3 was upregulated in migrating and metastatic tumor cells and was correlated with NSCLC prognosis. We found that SLC38A3 promoted metastasis of NSCLC cells through activation of PDK1/AKT signaling, suggesting SLC38A3 could be a potential therapeutic target for NSCLC treatment. 


\section{Materials and methods}

\subsection{Reagents and antibodies}

The following antibodies were purchased from Cell Signaling Technology (MA, USA) and used at the indicated dilution for western blot analysis: phospho-S6K (\#9234, 1:1000), S6K (\#9202, 1:1000), phospho-4EBP1 (\#2855, 1:1000), 4EBP1 (\#9452, 1:000), mTOR (\#2983, 1:1000), E-Cadherin (\#3195S, 1:1000), N-Cadherin(\#13116S, 1:500), and Vimentin (\#5741S, 1:5000), Akt(\#4691P,1:1000), Phospho-Akt (\#2965P,1:2000), Phospho-GSK-3ß (\#9323P, 1:1000), GSK-3 $\beta$ (\#9315L,1:1000). The SLC38A3/SNAT3 antibody for Western blot, (\#373705, 1:200) and $\beta$-actin (\#47778, 1:2000) were obtained from Santa Cruz Biotechchnology (Santa Cruz, CA, USA). SLC38A3 for IHC (\#14315-1-AP, 1:1000) was from ProteinTech (Rosemont, IL, USA). MK2206 (\#S1078) was from Selleck (Shanghai, China). Puromycin was obtained from Invitrogen (Shanghai, China). DNA polymerase kit and Restriction Enzymes were from Fermentas (Ontario, Canada).

\subsection{Cell culture}

Cell lines were maintained in media including $10 \%$ fetal bovine serum (FBS, PAA Laboratories, $\mathrm{GmbH}$, Germany) at $37^{\circ} \mathrm{C}$ and a $5 \% \mathrm{CO}_{2}$ atmosphere. $\mathrm{H} 1650, \mathrm{H} 358, \mathrm{Hcc} 827$, and $\mathrm{H} 1299$ human lung cancer cell lines were obtained from the cell bank of the Chinese Academy of Science (CAS,

Shanghai, China) and cultured in RPMI 1640 medium (Gibco/Life Technologies, Grand Island, NY, USA) supplemented with penicillin (100 units/ml; Life Technologies) and streptomycin (100 
units/ml, Life Technologies). A549 adenocarcinomic human alveolar basal epithelial cells and 293T human embryonic kidney cells (CAS) were cultured in high glucose Dulbecco's minimum essential Eagle medium (DMEM Gibco, USA). IMRT and WIT (CAS) were cultured in minimum essential medium (MEM, Gibco).

\subsection{Establishment of stable cell lines overexpressing SLC38A3}

Open reading frame SLC38A3 plasmids were obtained from Jikai Gene (Shanghai, China), and primers for SLC38A3 cloning were: SLC38A3: forward: 5'TTTGAATTCATGGATTACAAGGATGACGACGATAAGGAGGCGCCTTTGCAGACAGAG3'; reverse: 5'-TTTGGATCCCTAGTGGTTTCCTCCATGCCGGCT-3'. SLC38A3 OE cells were

constructed by lentivirus transfection. Approximately $6 \times 10^{5}-8 \times 10^{5} 293 \mathrm{~T}$ cells were plated in 60 $\mathrm{mm}$ culture dishes and transfected with $24 \mu \mathrm{l}$ polyethylenimine (PEI) and $8 \mu \mathrm{g}$ of vectors (plenti/SLC38A3-OE:psPAX2:pMD2G = 4:2:1) when grown to $80 \%$ confluence. Fresh medium was added $6 \mathrm{hrs}$ after transfection, and then collected $72 \mathrm{hrs}$ later. Exponentially growing A549/HCC827 cells were plated in 6-well dishes and rinsed twice with ice-cold phosphate buffered salime (PBS), then put the mixture of cell supernatant and 10\% FBS cell media (1:1) in the dishes and replaced with fresh media after $12 \mathrm{hrs}$. Finally the positive transfected cells were selected by puromycin after $72 \mathrm{hrs}$.

\section{4 cDNA synthesis and real time-PCR}

To assay the expression of SLC38A3 ,total RNA was extracted from A549/HCC827 (CONTROL) and SLC38A3 OE A549/HCC827 cells, and cDNA was reverse transcribed using an RT reagent 
kit (TAKARA, Dalian, China). After cDNA synthesis, quantitative real time PCR (qPCR) was performed with an ABI7500 real time PCR system (Life Technologies, Grand Island, NY, USA) using SYBR Green mixture. The primer sequences for cDNA qPCR were: SLC38A3, forward 5'-ATCTCCAACCTGTCCATCGC-3' and reverse 5'-GCCAACGGCAATAAGCACAT-3'. $\beta$-actin, forward 5'-GCGGGAAATCGTGCGTGACATT-3' and reverse 5'-GATGGAGTTGAAGGTAGTTTCG-3'. The Ct values of SLC38A3 were normalized to that of $\beta$-actin, which was used as an internal control.

\subsection{Ex vivo 4D model}

The Ex Vivo 4D model was created as previously described [21]. Briefly, all the cells were removed from rat lung with SDS and Triton-X, the acellular lungs were inoculated by tracheal infusion of 25 million A549, H1299, 393P, or 344SQ cells diluted in $50 \mathrm{~mL}$ of complete medium. The lungs were placed in a bioreactor with a pulmonary artery cannula connected to a pump and oxygenator. The media in the bioreactor flowed through the pulmonary artery at $6 \mathrm{cc} / \mathrm{min}$ for 24 hours before replacement with fresh media daily for 15 days. Circulating tumor cells (CTCs) were isolated by centrifugation ( $500 \mathrm{~g}$ for $5 \mathrm{~min}$ ) of media that had circulated through the reactor for 24 hours. The supernatant was removed, and the precipitated cells were resuspended in $100 \mu 1$ of complete media. The CTCs were counted and used in subsequent procedures or stored in Trizol (5 PRIME, Gaithersburg, MD, USA) at $-80^{\circ} \mathrm{C}$ until needed. We used trypan blue staining to count the live cells. Experiments were repeated three times.

\subsection{Experimental metastatic lung cancer model}


Four-week-old BALB/c nude mice were purchased from the Shanghai Animal Laboratory. The animals were handled following a protocol approved by the Institutional Animal Care and Use Committee of the Shanghai Jiao Tong University School of Medicine. Experimental metastatic tumors were obtained following injection of $1 \times 10^{6}$ cells into the tail vein. Metastatic cancer cells were obtained from lung tumors isolated from mice sacrificed 6 weeks after injection of tumor cells.

\subsection{Western blotting and immunohistochemical staining}

Cells were harvested and lysed on ice for $10 \mathrm{~min}$ in buffer containing $150 \mathrm{mM} \mathrm{NaCl}, 0.1 \%$ SDS, $1 \% \mathrm{NaMoO}_{4}, 1 \% \mathrm{NP} 40,50 \mathrm{mM}$ Tris- $\mathrm{HCl}(\mathrm{PH} 7.5)$, and $0.02 \% \mathrm{NaN}_{3}$ supplemented with $1 \mathrm{mM}$ phenylmethanesulfonyl fluoride (PMSF). Total protein concentration was determined with a bicinchoninic acid (BCA) assay kit (Ding Guo Biotechnology, Shanghai, China). Proteins were separated on polyacrylamide gels and transferred to nitrocellulose membranes. The membrane was separately incubated with antibodies against S6K, phospho-specific S6K, mTOR, E-Cadherin, N-Cadherin, or SLC38A3/SNAT3, with anti- $\beta$-Actin as an internal control.

Immunohistochemical staining of surgical specimens NSCLC specimens from tumors at various clinical stages was performed in $4 \mu \mathrm{m}$ serial sections of formalin-fixed, paraffin-embedded tissue. After deparaffinization, slides were placed in Epitope Retrieval Solution (citrate buffer 0.01M, $\mathrm{pH}$ 6.0) and heated in a microwave oven for 5 minutes. After cooling and washing with PBS, endogenous peroxidase was blocked by $0.3 \% \mathrm{H}_{2} \mathrm{O}_{2} /$ methanol for 10 minutes and incubated with $10 \%$ goat serum to block nonspecific binding of antibodies. The slides were then incubated with 
SLC38A 3 primary antibody at $4{ }^{\circ} \mathrm{C}$ overnight, followed by biotin conjugated secondary antibody and streptavidin horseradish peroxidase (HRP), 300 minutes each. Antigen-antibody complexes were visualized in DAB (3,3'-diaminobenzidine), cells were stained with hematoxylin, and then dehydrated and mounted. All incubation steps were done at room temperature. Cells were photographed and the images were analyzed with Image $\mathbf{J}$ software (https://imagej.nih.gov/ij/docs/index.html).

\subsection{Scratch test and Transwell migration assay}

A549/HCC827 and SLC38A3 OE A549/HCC827 cells were seeded in six-well plates and monolayers were gently scratched with a sterile $200 \mu \mathrm{l}$ pipette tip when they had grown to $90 \%$ confluence. After washing with PBS three times to remove floating cells, the remaining adherent cells with a thin "wound" were incubated at $37^{\circ} \mathrm{C}$ in $5 \% \mathrm{CO}_{2}$. The photos were photographed at 0、12、24、36, $48 \mathrm{~h}$ using an inverted microscope. All independent experiments were run in triplicate.

The Transwell migration assay was carried out to assay the invasiveness of SLC38A3 OE cells.

Cultured cells were trypsinized, and suspended at a final concentration of $1 \times 10^{5}$ cells $/ \mathrm{ml}$ in culture medium, seeded into the upper chamber in 24-well plates and cultured for $12 \mathrm{~h}$. After incubation, the transwell chambers were placed into new 24-well plates. washed with PBS and fixed. Non-migratory cells were removed with a cotton swab, and the remaining cells were 
stained with hematoxylin and eosin and counted using an inverted microscope. Photos of three random fields were taken.

\subsection{Mass spectrometry of metabolites}

The cellular amino acids content was analyzed by gas chromatography-mass spectroscopy (GC-MS) as previously described [22]. Cultured cells $\left(1 \times 10^{8}\right)$ were harvested and suspended in chloroform-methanol-water (2:1:1, v:v:v). The derivatized sample was injected into a Shimadzu QP 2010 GC tandem quadrupole MS (Kyoto, Japan). The GC separation was performed on an Agilent DB-5 MS fused silica capillary column $(30 \mathrm{~m} \times 0.25 \mathrm{~mm} \times 0.25 \mu \mathrm{m})$. The column temperature was $70{ }^{\circ} \mathrm{C}$ for the first $3 \mathrm{~min}$ and then increased at $5{ }^{\circ} \mathrm{C} / \mathrm{min}$ to $310{ }^{\circ} \mathrm{C}$ for $5 \mathrm{~min}$. The injection temperature was set at $300^{\circ} \mathrm{C}$, and the injection volume was $1 \mu \mathrm{L}$ with a $10: 1$ split ratio. Helium (99.9995\%, China) was used as the carrier gas. The column flow was $1.2 \mathrm{~mL} / \mathrm{min}$; the column was equipped with a linear velocity control. The mass spectra scanning analyzer was set to $33-600 \mathrm{~m} / \mathrm{z}$ in the full scan mode with a scan speed of 5 scans s-1 and a solvent cut time of 5.6 min based on the retention time of the pyridine solvent. The temperatures of the interface and the ion source were adjusted to $280^{\circ} \mathrm{C}$ and $240^{\circ} \mathrm{C}$, respectively. The detector voltage was maintained at $1.2 \mathrm{kV}$, and the electron impact (EI) model was selected to achieve ionization of the metabolites at $70 \mathrm{eV}$.

\subsection{Statistical analysis}


The animal data are reported as medians $\pm \mathrm{SD}$; other data are reported as means $\pm \mathrm{SD}$. All data are representative of at least three independent experiments. The differences between groups were assessed by Student's $t$ test; all reported significant differences were $\mathrm{p}<0.05$ unless otherwise stated. 


\section{Results:}

\subsection{SLC38A3 expression is up-regulated in metastatic NSCLC cells}

Circulating cancer cells are, seeds for metastatic tumor formation, making them perfect cells for study of metastatic cancer cell characteristics. The 4D lung cancer model [21] is ideally suited for studying tumor metastasis as tumor cells can be easily isolated in three phases of tumor progression, primary, circulating and metastatic. To help identify metabolic change(s) involved in regulating metastasis of NSCLC, transcriptome array data (Access number: GSE58355) were searched for expression of metabolism-related genes in the metastatic cancer cells, circulating cancer cells, and the primary cancer cells in the 4D lung cancer model. As shown in Table 1 and Figure 1A, among members of APC superfamily, only glutamine transporter SLC38A3, and no other member of the SLC38, SLC32 or SLC36 family (Figure S1A) was upregulated in both circulating and metastatic NSCLC cells, compared with primary cancer cells, suggesting SLC38A3 is associated with tumor metastasis.

To determine whether the SLC38A3 was involved in NSCLC progression, the expression of SLC38A3 protein was analyzed by histoimmunochemical staining on a tissue array of clinical NSCLCs. As shown in Figure 1B, SLC38A3 expression was significantly upregulated in all NSCLC tumors compared with the paratumor tissue. Further analysis showed that SLC38A3 expression was relatively higher in metastatic than primary NSCLC tumor cells (Figure 1C). 
To further investigate whether the expression of SLC38A3 was correlated with NSCLC cell

metastasis of, SLC38A3 protein expression was assayed in an experimental nude mouse model of A549 cells metastasis following tail vein injection. The morphology of metastatic A549 cells verified by green fluorescent protein (GFP, Figure S1B) was evaluated. As shown in Figure 1D, the morphology of metastatic A549 cells appeared mesenchymal, compared with primary A549 cells, further confirming the successful establishment of the experimental metastasis model. The western blots in Figure 1D show that SLC38A3 expression was significantly increased in metastatic, compared with primary, A549 cells, suggesting SLC38A3 may be involved in the metastasis of NSCLC.

\subsection{SLC38A3 promotes EMT and migration of NSCLC cells}

To determine whether SLC38A3 promoted NSCLC metastasis, we first assayed its expression in the available cell lines. As shown in Figure 2A, SLC38A3 expression was upregulated in NSCLC cells compared with normal lung epithelium, which was consistent with the clinical data. Moreover, the overexpression of SLC38A3 was associated with a change of the epithelium-like morphology of A549 cells to a mesenchyme-like morphology, similar to that of TGF- $\beta$-treated A549 cells, which were a positive control (Figure 2B). SLC38A3 overexpression was associated with a similar change in HCC827 NSCLC cells (Figure S2A).

To determine whether SLC38A3 promoted the EMT of NSCLC cells, we assayed the expression of two EMT markers, E-cadherin and N-cadherin, by western blotting or immunofluorescence 
staining. The western blot results showed that both overexpression of SLC38A3 and TGF- $\beta$

treatment decreased the expression of E-cadherin (epithelial marker) and increased the expression of N-cadherin (a mesenchymal marker) in A549 cells (Figure 2C) and HCC827 cells (Figure S2B). The results of immunofluorescence staining were consistent with the western blot assays (Figures 2D and S2C). These observations suggest SLC38A3 promoted the EMT of NSCLC cells.

To determine whether SLC38A3 promoted metastasis of NSCLC, the scratch test and the Transwell migration assay were performed using A549 and HCC827 NSCLC cells. As shown in Figures 2E and S2D, overexpression of SLC38A3 increased the migration rate of both A549 cells and HCC827 cells, compared with their control cells. The Transwell migration assay also showed that overexpression of SLC38A3 increased the migration rate of both cell lines (Figures 2F and S2E). These observations suggest that SLC38A3 promoted migration of NSCLC cells.

\subsection{SLC38A3 activates PDK1/AKT signaling by decreasing the cellular concentration of}

\section{glutamine/histidine}

To determine the mechanism underlying SLC38A3 promotion of NSCLC metastasis, the activation of three pathways that regulate EMT was investigated in A549 cells. As shown in Figure 3A, overexpression of SLC38A3 increased the phosphorylation of AKT at Thr 308, and activated AKT/GSK $3 \beta$ but not Smad2/3 or ERK/STAT3 signaling. The activation of AKT/GSK3 $\beta$ 
signaling ultimately increased the expression of Snail, which is a transcription factor that promotes the repression of E-cadherin.

To test whether the AKT signaling mediated by SLC38A3 regulated EMT, the expression of a critical EMT regulator Snail was assayed in A549 cells overexpressing SLC38A3. Compared to control group, the AKT inhibitor MK2206 significantly decreased the protein level of Snail (Figure 3B), which is consistent with inactivation of AKT/GSK3 $\beta / \beta$-catenin signaling. To further determine whether SLC38A3 regulated EMT by activating AKT, the epithelial marker E-Cadherin and the mesenchymal marker N-Cadherin were assayed by Western blot. As shown in Figure 3C, the AKT inhibitor MK2206 impaired SLC38A3 promotion of N-Cadherin expression and partially recovered SLC38A3 inhibition of E-Cadherin expression. This suggests that SLC38A3 promoted EMT via the AKT/GSK3 $\beta / \beta$-catenin pathway.

Since SLC38A3 increased the Thr 308 phosphorylation of AKT, the activity of PDK1, its upstream kinase, was assayed. Compared with A549 control cells, overexpression of SLC38A3 significantly increased PDK1 phosphorylation at Ser 241, which reflected the activation of PDK1 (Figure 3D). To explore the mechanism by which SLC38A3 activated PDK1, the cellular amino acid concentration was analyzed by GC-MS with SLC38A3 as an amino acid carrier. As shown in Figure 3E, overexpression of SLC38A3 significantly decreased the concentration of glutamine and histidine $(\mathrm{p}<0.01)$, but not alanine or aspartate, in NSCLC A549 cells. The depletion of glutamine or histidine activated PDK1, and the Thr 308 phosphorylation of AKT increased along 
with this activation, suggesting that overexpression of SLC38A3 activated PDK1 by decreasing the cellular concentration of glutamine and histidine (Figure 3E).

\subsection{SLC38A3 promotes metastasis of NSCLC A549 cells in mice}

To further investigate whether SLC38A3 promoted metastasis of NSCLC cells in vivo, control A549 or A549 cells overexpressing SLC38A3 were injected into nude mice. As shown in Figure 4A, A549 cells successfully migrated into the lungs from the circulation, and overexpression of SLC38A3 promoted lung cancer formation (15 tumors vs. 5 tumors per mouse), suggesting SLC38A3 promoted metastasis.

Immunohistochemical staining of formalin-fixed paraffin-embedded metastatic tumors confirmed the expression of phosphorylated AKT in cells that also expressed SLC38A3, as shown in Figure 4B. The immunohistochemical staining was consistent with SLC38A3 promotion of AKT activation that in turn triggered epithelial-mesenchymal transition of NSCLC cells. Finally, the effect of SLC38A3 expression on prognosis of NSCLC patients was estimated by the Kaplan-Meier method. As shown in Figure 4C, low expression of SLC38A3 (in 968 of 1926 NSCLC patients) prolonged survival. High expression shortened survival. These observations suggest that SLC38A3 as a potential therapeutic target for NSCLC. 


\section{Discussion}

Mammalian target of rapamycin (mTOR), a conserved Ser/Thr kinase, is a master regulator that couples amino acid availability to cell growth and autophagy. Although numerous studies have demonstrated that increased cellular amino acids trigger mTORC1 activation in a tuberous sclerosis complex (TSC)-dependent or TSC-independent manner [23; 24; 25]. It is not clear which signaling pathway is activated in response to the absence of specific amino acids. Our data have shown that glutamine /histidine efflux induced PDK1 activation and subsequent AKT phosphorylation at Thr 308. This is a novel mechanism of AKT activation, suggesting that PDK1 may be a sensor for the absence of glutamine/histidine and a distinct response of tumor cells to a specific change in amino acid content.

Previous studies demonstrated that glutamate/glutamine and glucose metabolism both play key roles in metabolic reprogramming, and some indicated that glutamine/glutamate metabolism might regulate tumor metastasis $[11 ; 12]$. In this study, we demonstrated that overexpression of the solute carrier SLC38A3 increased glutamine/histidine export and activated AKT/GSK3 $\beta / \beta$-catenin signaling, which, in turn induced EMT and metastasis of NSCLC cells. Once tumor cells detach from the primary site, the $\mathrm{H}^{+}$gradient is reversed, leading to glutamine efflux and PDK1 activation. These observations are in line with evidence that changes in the microenvironment regulate tumor cell behavior by modulating tumor cell metabolism. 
SLC38 transporters undoubtedly have many physiological roles, including transfer of glutamine from astrocytes to neurons in the CNS, ammonia detoxification and gluconeogenesis in the liver, and the renal response to acidosis [17]. System A subtypes (SNAT1, SNAT2, and SNAT4) transport small, aliphatic amino acids and are rheogenic and $\mathrm{pH}$ sensitive, whereas system $\mathrm{N}$ subtypes including SLC38A3 (SNAT3) and SLC38A5 (SNAT5) mediate the cotransport of $\mathrm{Na}^{+}$ with neutral amino acids in exchange for a $\mathrm{H}^{+}$. System $\mathrm{N}$ subtypes also counter transport $\mathrm{H}^{+}$, but have narrower substrate profiles than do the System A subtypes. Glutamine emerges as a favored substrate throughout most of this family [18]. A change in the $\mathrm{pH}$ of a tumor niche may induce the upregulation of SLC38A3 in circulating tumor and metastatic cells, compared with cells in the primary tumor. Recent studies have revealed additional functions SLC38 transporters and have proposed them potential therapeutic targets in neoplasia [26; 27], which is consistent with our findings.

Briefly, we demonstrated that SLC38A3 was upregulated in metastatic tumor cells and that overexpression of SLC38A3 promoted EMT and migration of NSCLC cells by activating PDK1/AKT signaling. The results support additional study of SLC38A3 as a potential therapeutic target for NSCLC. 


\section{Ethical approval}

Experimental animals were handled following the protocol approved by the Institutional Animal Care and Use Committee of the Shanghai Jiao Tong University School of Medicine.

\section{Consent for publication}

Not applicable.

\section{Availability of data and material}

All data generated or analyzed during this study are included in this published article.

\section{Conflict of Interest Disclosure}

All authors state that there are no conflicts of interest to disclose.

\section{Author contribution statement}

Y. W., L. F. and M. C. performed most of the experiments; Y. W. performed the animal experiments; X. L. provided reagents and revised the manuscript; J. M. designed the study and wrote the manuscript; all authors reviewed the manuscript.

\section{Acknowledgements}

We are grateful to Dr. Jinke Cheng, and Dr. Xuemei Tong (Shanghai Jiao Tong University School of Medicine) for valuable discussions.

\section{Funding source}

This study was supported by the Shanghai Committee of Science and Technology (grants number 
11DZ2260200), and the National Science Foundation of China (grant numbers 81372194, 81572300). 


\section{Reference:}

[1]G.F. Weber, Metabolism in cancer metastasis. International journal of cancer 138 (2016) 2061-2066.

[2]Z.T. Schafer, A.R. Grassian, L. Song, Z. Jiang, Z. Gerhart-Hines, H.Y. Irie, S. Gao, P. Puigserver, J.S. Brugge, Antioxidant and oncogene rescue of metabolic defects caused by loss of matrix attachment. Nature 461 (2009) 109-113.

[3]J. Folkman, E. Merler, C. Abernathy, G. Williams, Isolation of a tumor factor responsible for angiogenesis. J Exp Med 133 (1971) 275-288.

[4]G.F. Weber, Molecular mechanisms of metastasis. Cancer letters 270 (2008) 181-190.

[5]S. Kamarajugadda, L. Stemboroski, Q. Cai, N.E. Simpson, S. Nayak, M. Tan, J. Lu, Glucose oxidation modulates anoikis and tumor metastasis. Mol Cell Biol 32 (2012) 1893-1907.

[6]W. Liu, B.H. Beck, K.S. Vaidya, K.T. Nash, K.P. Feeley, S.W. Ballinger, K.M. Pounds, W.L. Denning, A.R. Diers, A. Landar, A. Dhar, T. Iwakuma, D.R. Welch, Metastasis suppressor KISS1 seems to reverse the Warburg effect by enhancing mitochondrial biogenesis. Cancer Res 74 (2014) 954-963.

[7]R.J. DeBerardinis, A. Mancuso, E. Daikhin, I. Nissim, M. Yudkoff, S. Wehrli, C.B. Thompson, Beyond aerobic glycolysis: transformed cells can engage in glutamine metabolism that exceeds the requirement for protein and nucleotide synthesis. Proc Natl Acad Sci U S A 104 (2007) 19345-19350.

[8]R.J. DeBerardinis, J.J. Lum, G. Hatzivassiliou, C.B. Thompson, The biology of cancer: metabolic reprogramming fuels cell growth and proliferation. Cell metabolism 7 (2008) 11-20.

[9]K. Walczak, S. Deneka-Hannemann, B. Jarosz, W. Zgrajka, F. Stoma, T. Trojanowski, W.A. Turski, W. Rzeski, Kynurenic acid inhibits proliferation and migration of human glioblastoma T98G cells. Pharmacol Rep 66 (2014) 130-136.

[10]Z.C. Ye, J.D. Rothstein, H. Sontheimer, Compromised glutamate transport in human glioma cells: reduction-mislocalization of sodium-dependent glutamate transporters and enhanced activity of cystine-glutamate exchange. J Neurosci 19 (1999) 10767-10777.

[11]S.A. Lyons, W.J. Chung, A.K. Weaver, T. Ogunrinu, H. Sontheimer, Autocrine glutamate signaling promotes glioma cell invasion. Cancer Res 67 (2007) 9463-9471.

[12]S.A. van Lith, A.C. Navis, K. Verrijp, S.P. Niclou, R. Bjerkvig, P. Wesseling, B. Tops, R. Molenaar, C.J. van Noorden, W.P. Leenders, Glutamate as chemotactic fuel for diffuse glioma cells: are they glutamate suckers? Biochim Biophys Acta 1846 (2014) 66-74.

[13]M.S. Almen, K.J. Nordstrom, R. Fredriksson, H.B. Schioth, Mapping the human membrane proteome: a majority of the human membrane proteins can be classified according to function and evolutionary origin. BMC Biol 7 (2009) 50.

[14]M.A. Hediger, B. Clemencon, R.E. Burrier, E.A. Bruford, The ABCs of membrane transporters in health and disease (SLC series): introduction. Mol Aspects Med 34 (2013) 95-107.

[15]M. Punta, P.C. Coggill, R.Y. Eberhardt, J. Mistry, J. Tate, C. Boursnell, N. Pang, K. Forslund, G. Ceric, J. Clements, A. Heger, L. Holm, E.L. Sonnhammer, S.R. Eddy, A. Bateman, R.D. Finn, The Pfam protein families database. Nucleic Acids Res 40 (2012) D290-301. 
[16]P.J. Hoglund, K.J. Nordstrom, H.B. Schioth, R. Fredriksson, The solute carrier families have a remarkably long evolutionary history with the majority of the human families present before divergence of Bilaterian species. Mol Biol Evol 28 (2011) 1531-1541.

[17]B. Mackenzie, J.D. Erickson, Sodium-coupled neutral amino acid (System N/A) transporters of the SLC38 gene family. Pflugers Arch 447 (2004) 784-795.

[18]F.A. Chaudhry, R.J. Reimer, D. Krizaj, D. Barber, J. Storm-Mathisen, D.R. Copenhagen, R.H. Edwards, Molecular analysis of system $\mathrm{N}$ suggests novel physiological roles in nitrogen metabolism and synaptic transmission. Cell 99 (1999) 769-780.

[19]A. Broer, A. Albers, I. Setiawan, R.H. Edwards, F.A. Chaudhry, F. Lang, C.A. Wagner, S. Broer, Regulation of the glutamine transporter SN1 by extracellular $\mathrm{pH}$ and intracellular sodium ions. J Physiol 539 (2002) 3-14.

[20]F.A. Chaudhry, D. Krizaj, P. Larsson, R.J. Reimer, C. Wreden, J. Storm-Mathisen, D. Copenhagen, M. Kavanaugh, R.H. Edwards, Coupled and uncoupled proton movement by amino acid transport system N. The EMBO journal 20 (2001) 7041-7051.

[21]D.K. Mishra, K.L. Scott, J.M. Wardwell-Ozgo, M.J. Thrall, M.P. Kim, Circulating tumor cells from 4D model have less integrin beta 4 expression. J Surg Res 193 (2015) 745-753.

[22]E.C. Chan, P.K. Koh, M. Mal, P.Y. Cheah, K.W. Eu, A. Backshall, R. Cavill, J.K. Nicholson, H.C. Keun, Metabolic profiling of human colorectal cancer using high-resolution magic angle spinning nuclear magnetic resonance (HR-MAS NMR) spectroscopy and gas chromatography mass spectrometry (GC/MS). Journal of proteome research 8 (2009) 352-361.

[23]E.M. Smith, S.G. Finn, A.R. Tee, G.J. Browne, C.G. Proud, The tuberous sclerosis protein TSC2 is not required for the regulation of the mammalian target of rapamycin by amino acids and certain cellular stresses. J Biol Chem 280 (2005) 18717-18727.

[24]X. Long, S. Ortiz-Vega, Y. Lin, J. Avruch, Rheb binding to mammalian target of rapamycin (mTOR) is regulated by amino acid sufficiency. J Biol Chem 280 (2005) 23433-23436.

[25]Y. Sancak, T.R. Peterson, Y.D. Shaul, R.A. Lindquist, C.C. Thoreen, L. Bar-Peled, D.M. Sabatini, The Rag GTPases bind raptor and mediate amino acid signaling to mTORC1. Science 320 (2008) 1496-1501.

[26]T. Hatanaka, W. Huang, R.G. Martindale, V. Ganapathy, Differential influence of cAMP on the expression of the three subtypes (ATA1, ATA2, and ATA3) of the amino acid transport system A. FEBS Lett 505 (2001) 317-320.

[27]H. Varoqui, H. Zhu, D. Yao, H. Ming, J.D. Erickson, Cloning and functional identification of a neuronal glutamine transporter. J Biol Chem 275 (2000) 4049-4054. 


\section{Figure legend:}

Figure 1 SLC38A3 expression was correlated with NSCLC metastasis. A. SLC38A3 expression increased with progression from primary NSCLC tumor cells to circulating NSCLC cells, to metastatic NSCLC cells. The access number of the microarray data is GSE58355. ${ }^{* *}: p<0.01,{ }^{*}: p<0.05, n=6$. B. Immunohistobiochemical staining of SLC38A3 in NSCLC samples and their corresponding paratumor tissues. Tissue was obtained from 138 cases, $\left.{ }^{* *}: p<0.01\right)$. C. Immunohistobiochemistry staining of SLC38A3 in primary NSCLC samples (14 cases) and metastastic NSCLC tissues (21 cases). ${ }^{* *}: \mathrm{P}<$ 0.01. D. SLC38A3 expression in A549 cells isolated from the experimental metastatic tumors from nude mice. A549 cells were isolated from lung tumors following tail vein injection.

Figure 2 SLC38A3 promotes the epithelial-mesenchymal transition and migration

of NSCLC A549 cells. A. SLC38A3 expression analyzed by Western blot in normal lung epithelial and NSCLC cell lines. B. Overexpression of SLC38A3 altered A549 cells morphology from epithelial-like to mesenchymal-like. TGF- $\beta 1$ treated A549 cells were considered as positive control. C. \& D. E-cadherin and N-cadherin expression assayed in Western blots and by immunofluorescence staining. E. Cell migration assayed by the scratch test in A549 control cells or A549 cells overexpressing SLC38A3; ${ }^{\star *}: P<0.01$. F. 
Transwell assay of cell migration of A549 control cells or A549 cells overexpressing SLC38A3.

$* * \mathrm{P}<0.01$

\section{Figure 3 Overexpression of SLC38A3 activated PDK1/AKT signaling through}

glutamine/histidine export. A. Overexpression of SLC38A3 increased the T308 phosphorylation of AKT, but did not affect Smad 2/3 nor ERK/Stat signaling. B. The AKT Inhibitor MK2206

abolished SLC38A3-enhanced Snail expression in A549 cells. The final concentration of MK2206 was 5 $\mu$ M. C. The AKT Inhibitor MK2206 abolished SLC38A3-promoted EMT of A549 cells. D. Overexpression of SLC38A3 activated PDK1. E. Overexpression of SLC38A3 decreased the cellular concentration of glutamine and histidine. The cellular concentration of amino acids was determined by GC-MS. F. Depletion of glutamine or histidine triggered the activation of PDK1.

Figure 4. Overexpression of SLC38A3 promoted the metastasis of NSCLC A549 cells. A. Overexpression of SLC38A3 promoted lung metastasis of A549 cells. The experimental nude mouse metastasis model was established by tail vein injection of tumor cells. $* * \mathrm{P}<0.01$. B. overexpression of SLC38A3 increased AKT activation. Phosphorylated AKT and SLC38A3 detected by IHC staining on xenograft tumor. C. SLC38A3 expression was inversely correlated with prognosis in NSCLC patients. D. A proposed model of SLC38A3 promotion of PDK1 activation and NSCLC metastasis. 
Figure 1

A

\begin{tabular}{|c|c|c|c|}
\hline & Primary & Circulating & Metastatic \\
\hline SLC38A1 & 1404.37095 & 1354.1021 & 772.64695 \\
\hline SLC38A10 & 515.687 & 505.58525 & 406.35935 \\
\hline SLC38A11 & 14.54055 & 12.834 & 17.22665 \\
\hline SLC38A2 & 17594.09285 & 23336.7381 & 15409.3283 \\
\hline SLC38A3 & 138.5511 & 160.70455 & 232.3243 \\
\hline SLC38A4 & 1.8372 & 2.1644 & 3.68955 \\
\hline SLC38A5 & 13.37875 & 12.43125 & 16.86795 \\
\hline SLC38A6 & 235.5254 & 197.4416 & 286.1347 \\
\hline SLC38A8 & 138.60665 & 161.6408 & 127.72895 \\
\hline SLC38A9 & 244.6642 & 237.4065 & 193.33345 \\
\hline
\end{tabular}

B

$B$

Paratumor

Lung cancer
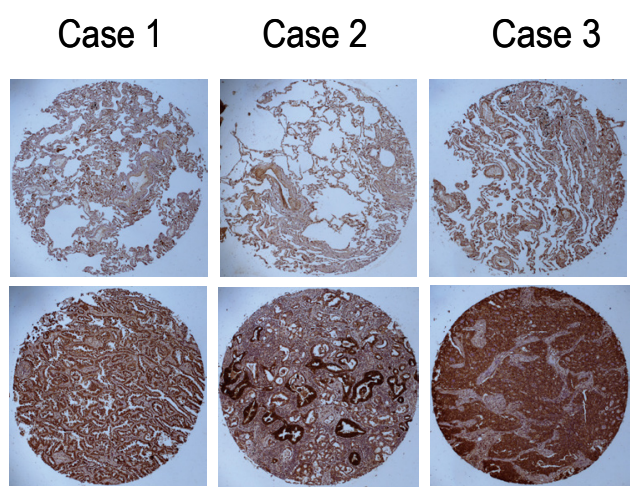

C
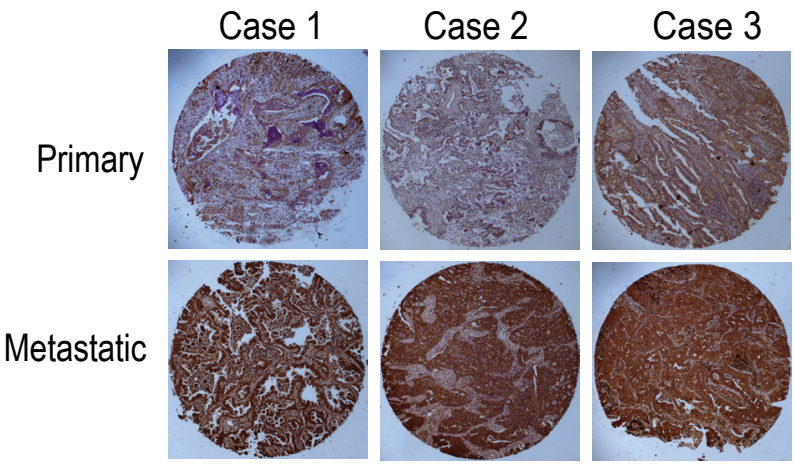

D

Primary
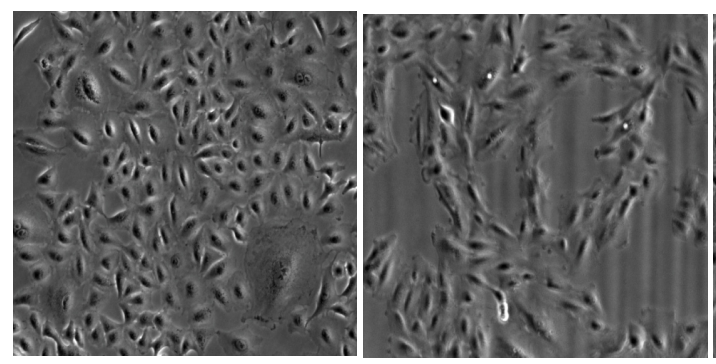

First

Second

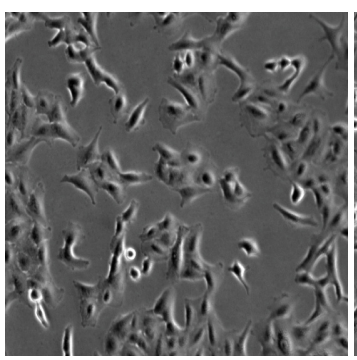

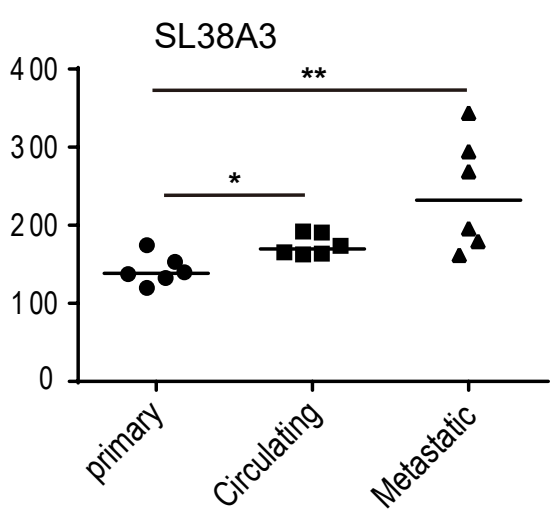
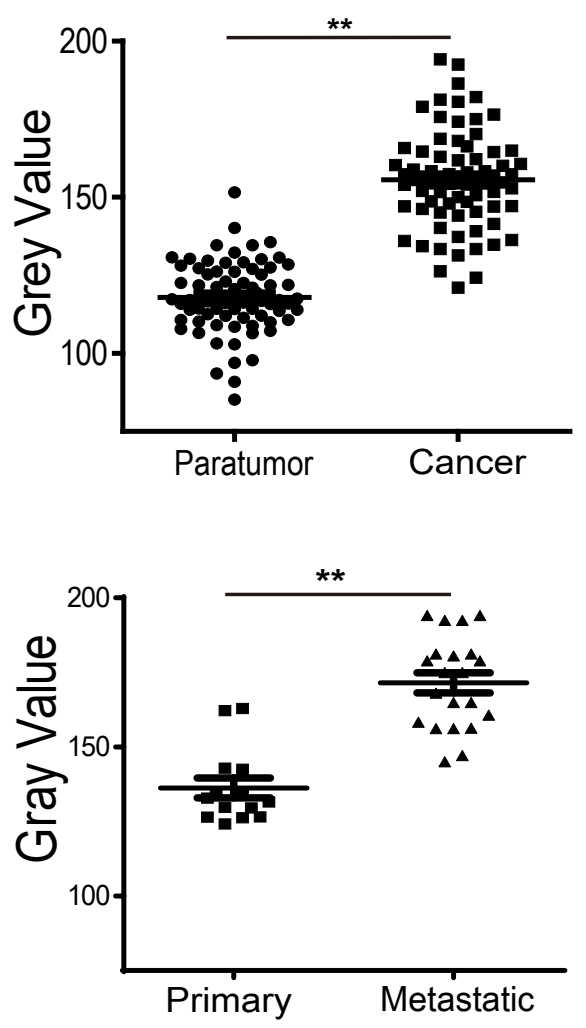

Third

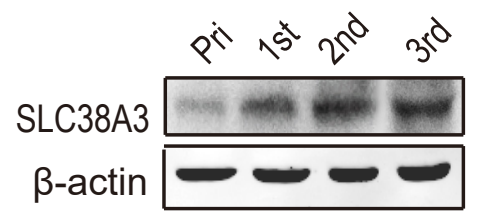


A

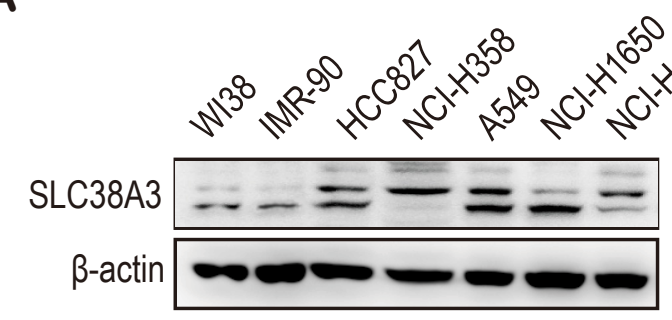

B

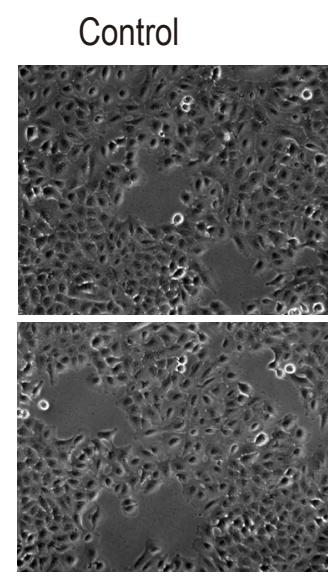

SLC38A3-OE
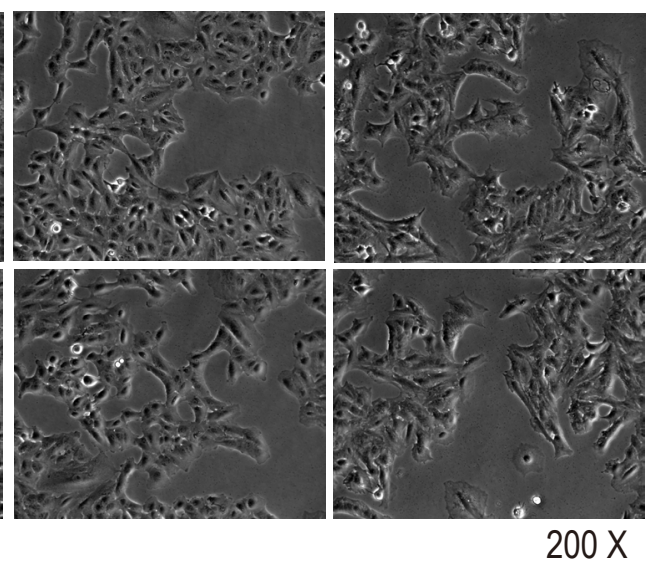

$200 X$
TGF- $\beta 1$

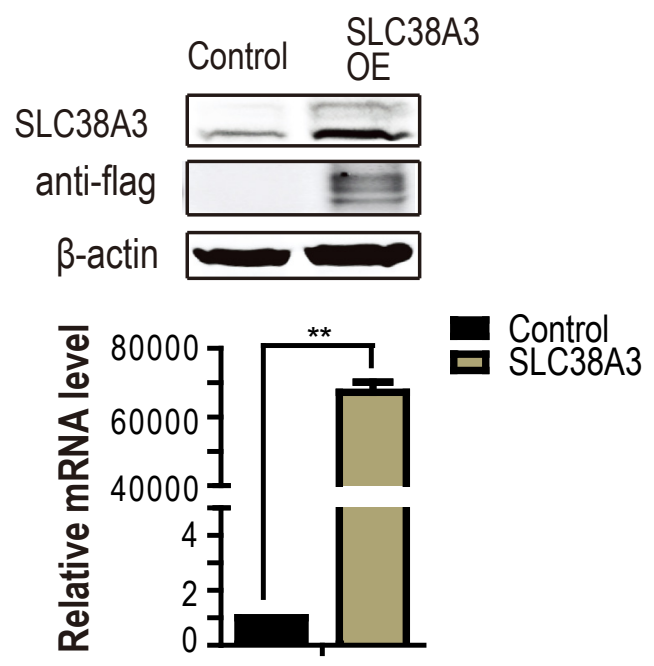

C

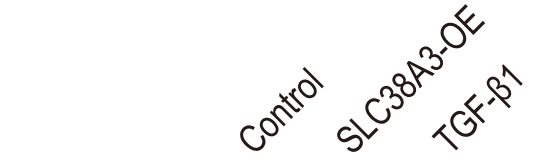

E-cadherin

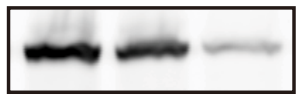

$\mathrm{N}$-cadherin

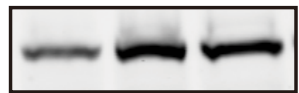

SLC38A3

$\beta$-actin

D

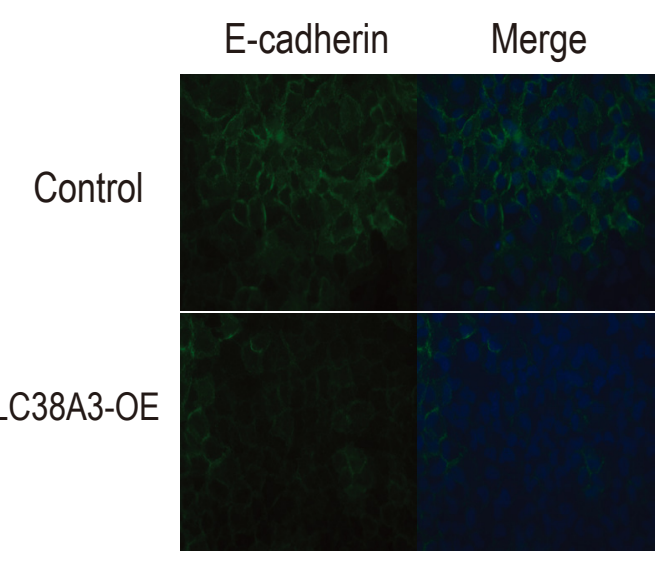

$\mathrm{N}$-cadherin

Merge

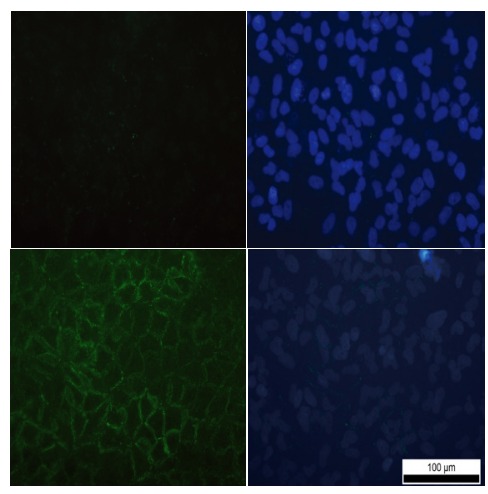

F

E

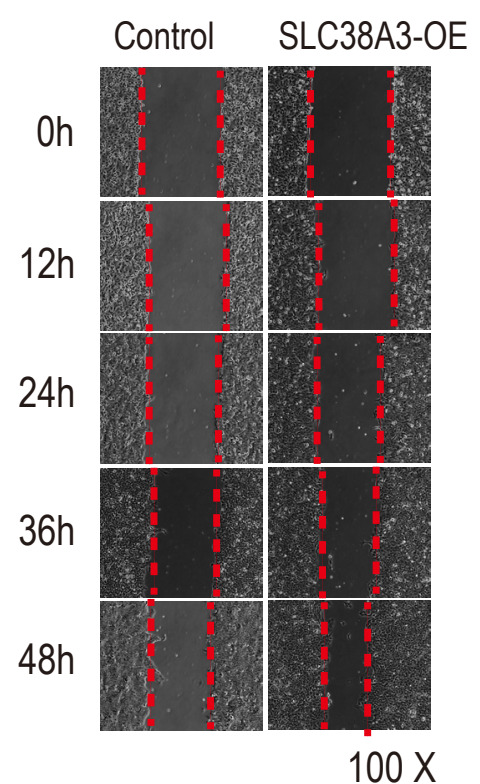

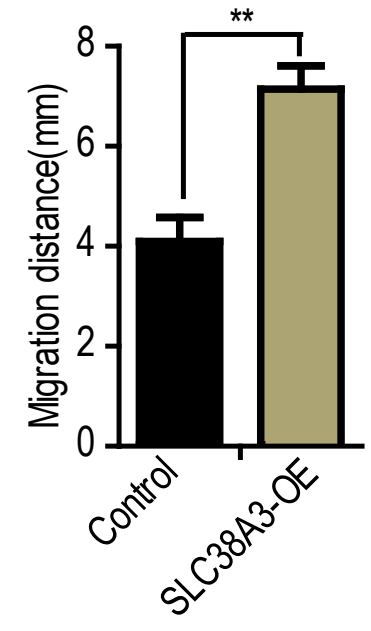
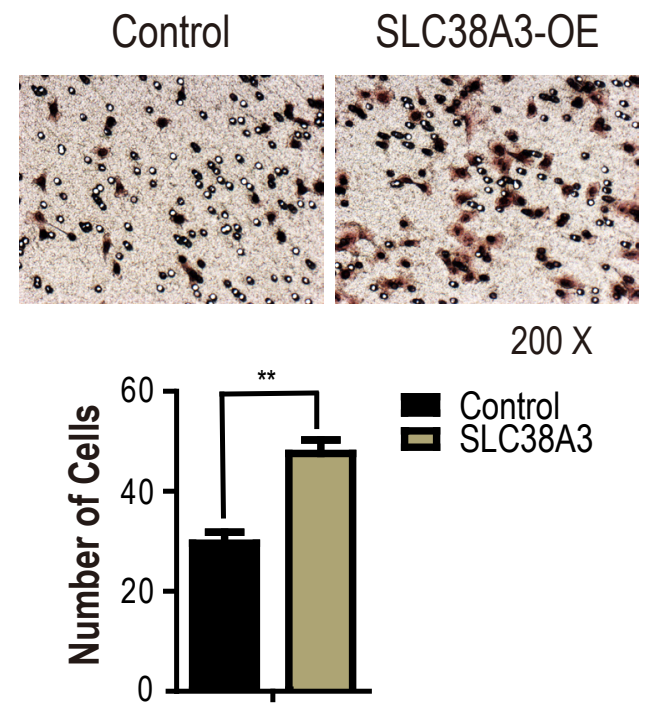
A
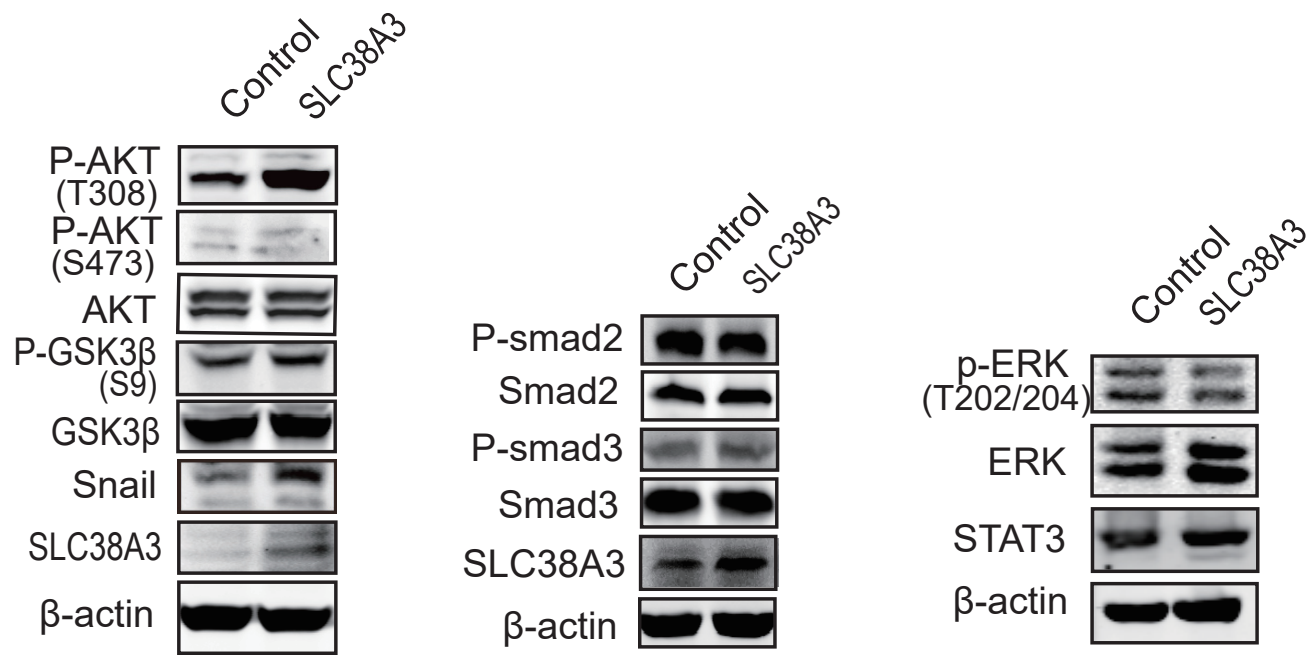

B

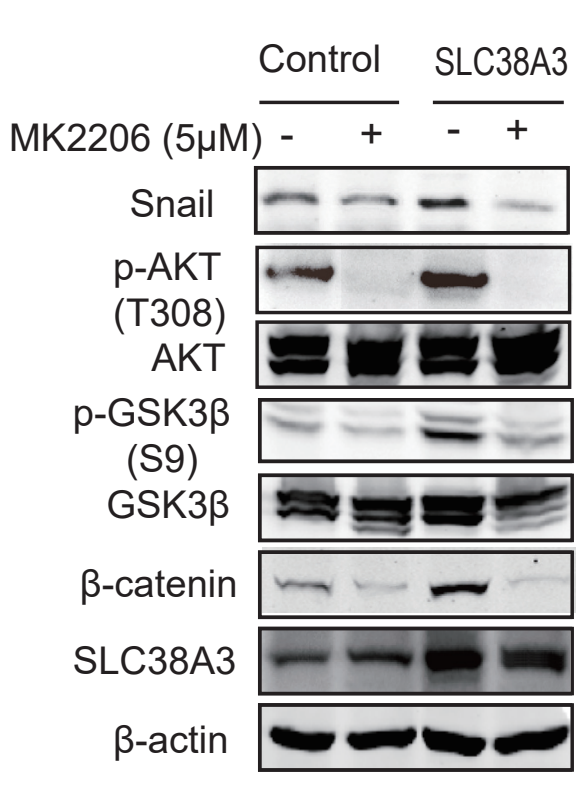

C

D

E
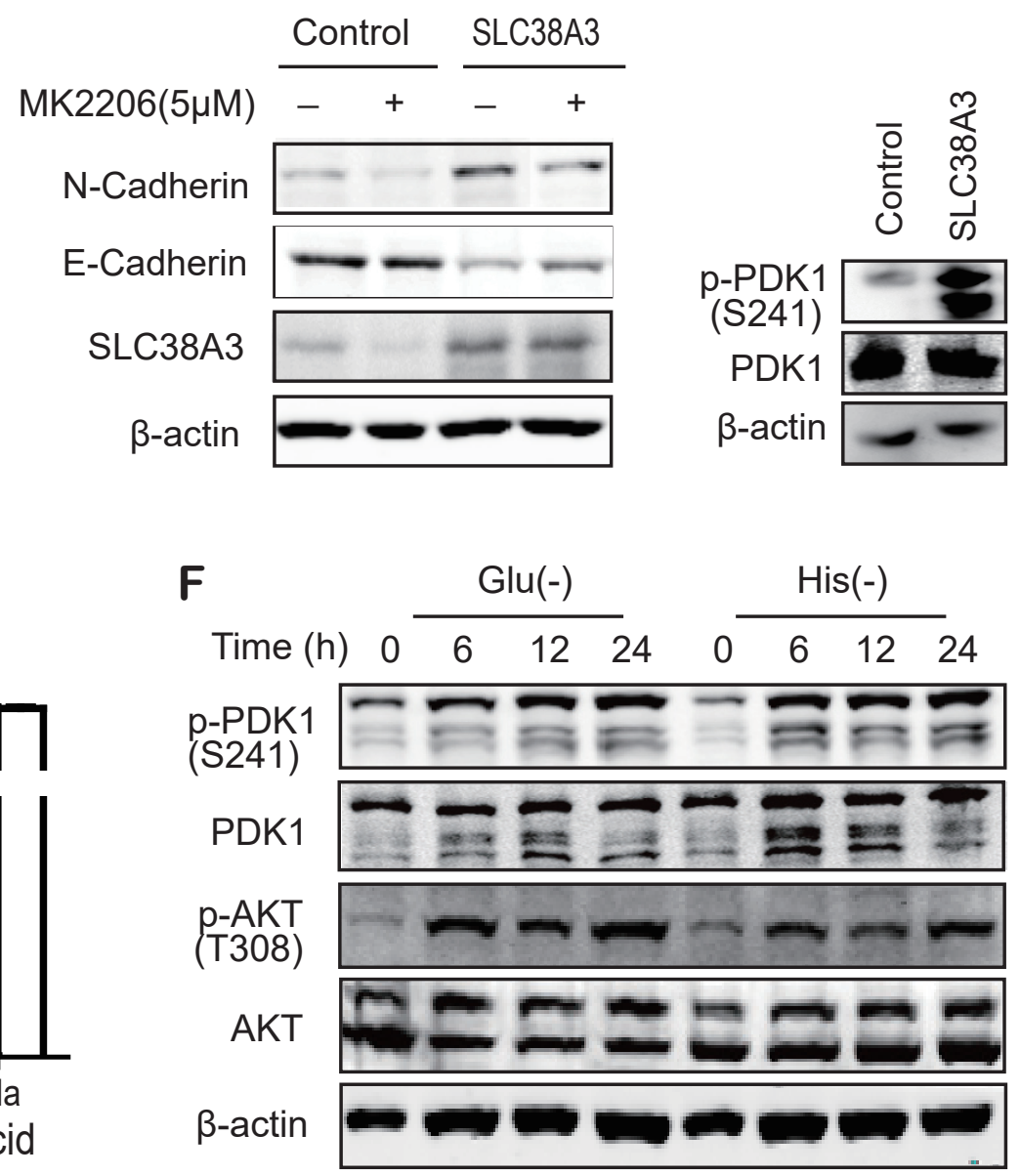
A
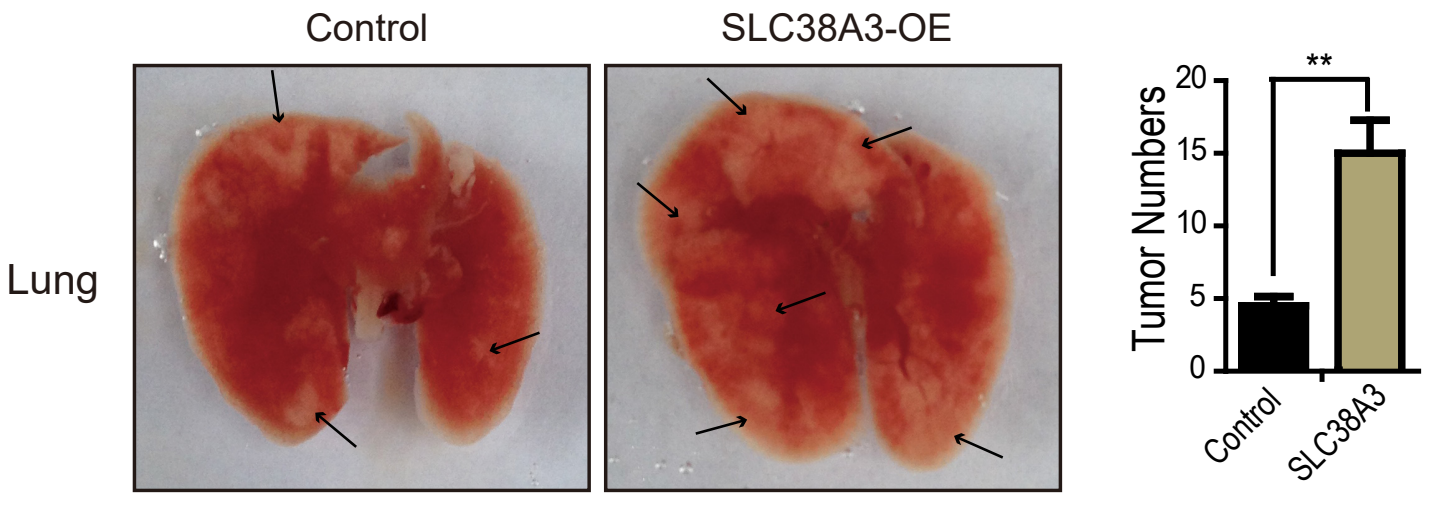

B
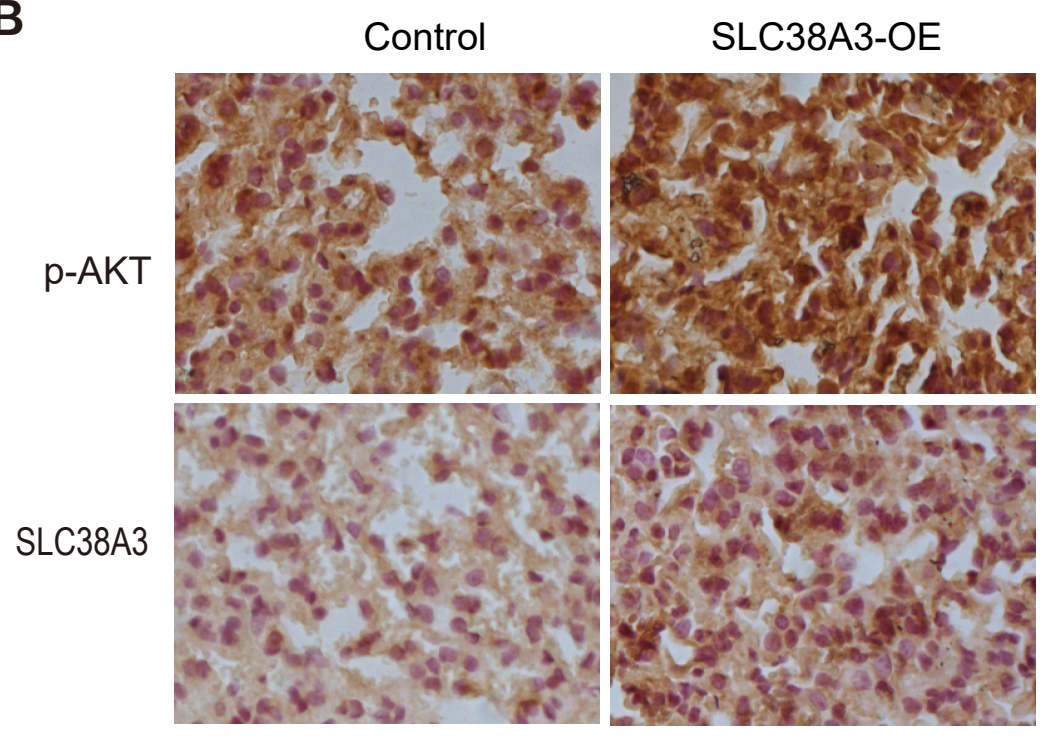

C

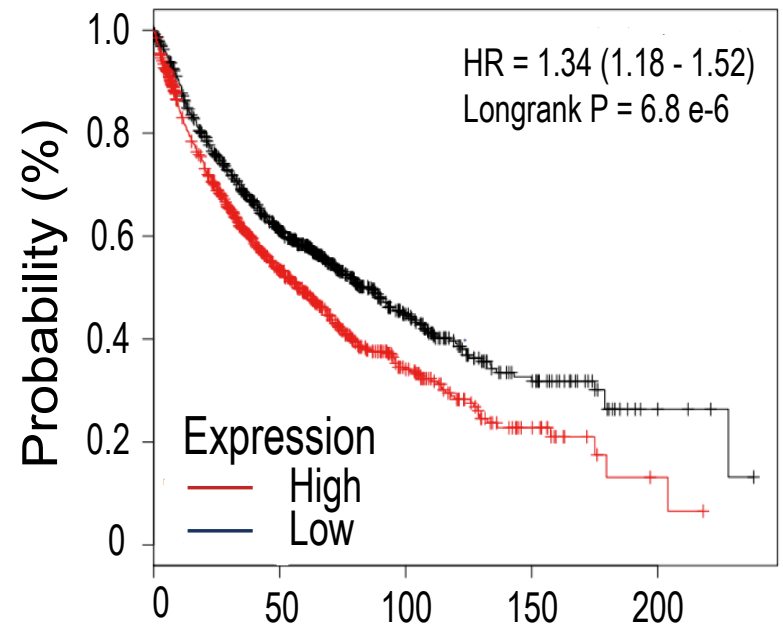

D

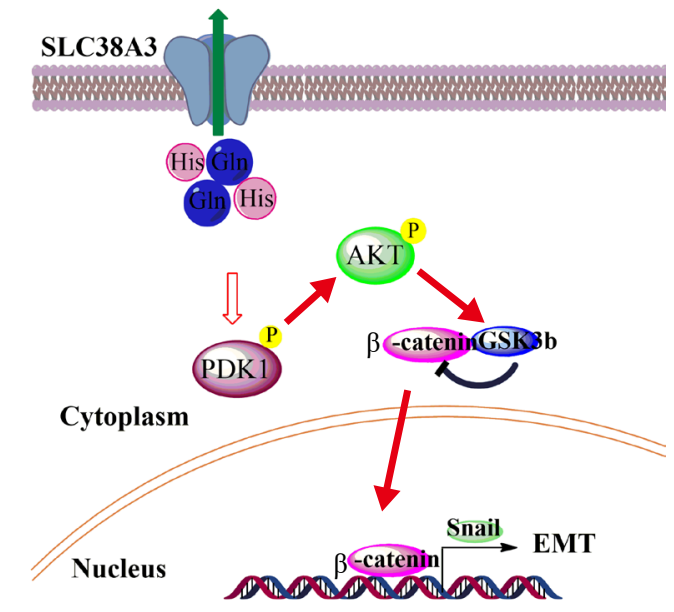

